\title{
Transcriptional activation of ENPP2 by FoxO4 protects cardiomyocytes from doxorubicin-induced toxicity
}

\author{
LING HE $^{1,2^{*}}$, YUTING YANG ${ }^{1 *}, \mathrm{JUAN} \mathrm{CHEN}^{2}$, PENGTAO ZOU $^{1}$ and JUXIANG LI ${ }^{1}$ \\ ${ }^{1}$ Department of Cardiovascular Medicine, The Second Affiliated Hospital of Nanchang University, Nanchang, Jiangxi 330006; \\ ${ }^{2}$ Department of Clinical Medicine, Jiangxi Health Vocational College, Nanchang, Jiangxi 330052, P.R. China
}

Received January 15, 2021; Accepted June 16, 2021

DOI: $10.3892 / \mathrm{mmr} .2021 .12307$

\begin{abstract}
It has been shown that ferroptosis is involved in doxorubicin (DOX)-induced cardiotoxicity and that ectonucleotide pyrophosphatase/phosphodiesterase 2 (ENPP2) can protect cardiomyocytes from ferroptosis. Thus, the present study aimed to investigate whether ENPP2 could protect cardiomyocytes from DOX-induced injury by inhibiting ferroptosis. H9c2 cardiomyocytes were exposed to various concentrations $(0.625,1.25,2.5,5$ or $10 \mu \mathrm{M})$ of DOX for different time periods. Cell viability and ENPP2 expression were determined. ENPP2-overexpressing H9c2 cells were treated with DOX and subsequently cell viability, oxidative stress, autophagy and ferroptosis were measured using the corresponding assays (MTT assay, commercial kits and western blot analysis). Dual-luciferase reporter and chromatin immunoprecipitation assays, as well as bioinformatics analysis, were applied to detect the interaction between ENPP2 and FoxO4. Following FoxO4 overexpression in $\mathrm{H} 9 \mathrm{c} 2$ cells, the aforementioned cellular processes were assessed. The results indicated that ENPP2 expression was downregulated following treatment of the cells with DOX. DOX also led to the decreased cell viability, reduced autophagy and elevated ferroptosis in H9c2 cells, which were notably reversed by ENPP2 overexpression. In addition, FoxO4 bound to the ENPP2 promoter, resulting in inhibition of its expression. Following FoxO4 overexpression in $\mathrm{H} 9 \mathrm{c} 2$ cells, further experiments conducted using commercial kits and western blot analysis revealed that FoxO4 overexpression partially inhibited the effects of ENPP2 overexpression on DOX-induced oxidative stress, autophagy and ferroptosis in $\mathrm{H} 9 \mathrm{c} 2$ cells. In conclusion, the data indicated that ENPP2 was transcriptionally regulated by FoxO4 to protect
\end{abstract}

Correspondence to: Dr Juxiang Li, Department of Cardiovascular Medicine, The Second Affiliated Hospital of Nanchang University, 1 Minde Road, Nanchang, Jiangxi 330006, P.R. China

E-mail: lijuxiangljx@163.com

${ }^{*}$ Contributed equally

Key words: cardiotoxicity, doxorubicin, ectonucleotide pyrophosphatase/phosphodiesterase 2, ferroptosis, FoxO4 cardiomyocytes from DOX-induced toxicity by inhibiting ferroptosis. Therefore, specific treatment approaches targeting the FoxO4/ENPP2 axis and ferroptosis may provide potential therapies for alleviating DOX-induced cardiotoxicity.

\section{Introduction}

Cardiovascular complications due to cancer therapy cause a significant reduction in treatment efficacy for patients with cancer (1). Doxorubicin (DOX) is a potent anthracycline that has been confirmed to cause long-term cardiovascular side effects and decrease the quality of life of cancer survivors, which eventually reduces the clinical application of DOX (2). Among the diverse mechanisms involved in the cardiotoxicity of DOX, the contribution of ferroptosis has been recently reported by an increasing number of studies (3-5).

Ferroptosis is a novel type of regulated cell death that is different from apoptosis and necrosis. It is an iron- and reactive oxygen species (ROS)-dependent cell death, characterized by the accumulation of ROS and inactivation of the cellular antioxidant glutathione (GSH), leading to redox dysregulation (6). Ferroptosis has been implicated in the pathological processes associated with carcinogenesis, degenerative diseases, stroke and kidney ischemia/reperfusion injury (7). Recently, ferroptosis was shown to exhibit a crucial role in DOX-induced cardiotoxicity. For instance, it has been documented that ferroptosis promoted DOX-induced cardiomyopathy and mortality in mice, whereas the ferroptosis inhibitor ferrostatin-1 and iron chelation ameliorate this process and effectively improve the survival rate of mice (4). In addition, mitochondrial ferroptosis has been shown to be a major cause of DOX-induced cardiotoxicity (2). It has also been reported that overexpression of GSH peroxidase 4 (GPX4) ameliorated cardiac impairment in mice, whereas GPX4 knockdown exacerbated this process (3). Therefore, inhibition of ferroptosis may effectively reduce DOX-induced cardiotoxicity.

Autotaxin (ATX) is a secreted enzyme encoded by the ectonucleotide pyrophosphatase/phosphodiesterase 2 (ENPP2) gene, which is important for generating lysophosphatidic acid (LPA). The ATX/LPA pathway serves a major role in embryonic, vascular and neuronal development (8). Disturbances in normal ATX/LPA signaling are associated with the development of multiple diseases, including cardiovascular disease (9). A previous study reported that ENPP2/LPA protected 
cardiomyocytes from erastin-induced ferroptosis, indicating the inhibitory effect of ENPP2 on ferroptosis induction in cardiomyocytes (10). The transcription factor FoxO4, which is a member of the FoxO transcription factor family, is predicted to bind to the promoter of ENPP2. The FoxO proteins are involved in a variety of biological processes, including cell proliferation, oxidative stress response, metabolism, immunity and apoptosis (11). It has been suggested that FoxO4 may be involved in aggravating cardiovascular diseases (12). FoxO4 was also reported to regulate DOX-induced toxicity in liver and kidney cells both in vivo and in vitro (13). In the present study, it was hypothesized that FoxO4 may be a transcriptional regulator of ENPP2 by inhibiting ferroptosis, which in turn can protect cardiomyocyte $\mathrm{H} 9 \mathrm{c} 2$ cells against DOX-induced injury.

\section{Materials and methods}

Cell culture and treatment. $\mathrm{H} 9 \mathrm{c} 2$ rat cardiomyocytes were purchased from The Cell Bank of Type Culture Collection of The Chinese Academy of Sciences and cultured in DMEM (Gibco; Thermo Fisher Scientific, Inc.) supplemented with 10\% FBS (Gibco; Thermo Fisher Scientific, Inc.) and 1\% penicillin-streptomycin (Gibco; Thermo Fisher Scientific, Inc.). The cells were incubated at $37^{\circ} \mathrm{C}$ in a humidified atmosphere containing $5 \% \mathrm{CO}_{2}$. The cells were exposed to $0.625,1.25$, 2.5, 5 or $10 \mu \mathrm{M}$ DOX (Sigma-Aldrich; Merck KGaA) at $37^{\circ} \mathrm{C}$ for 12,24 or $48 \mathrm{~h}$ to detect cell viability. Cells were treated with $0.625,1.25,2.5,5$ or $10 \mu \mathrm{M}$ DOX (Sigma-Aldrich; Merck $\mathrm{KGaA}$ ) for $24 \mathrm{~h}$ at $37^{\circ} \mathrm{C}$ or with $5 \mu \mathrm{M}$ DOX for 12,24 and $48 \mathrm{~h}$ at $37^{\circ} \mathrm{C}$ to determine the mRNA and protein expression of ENPP2. In subsequent experiments, $5 \mu \mathrm{M}$ DOX was used for $24 \mathrm{~h}$ to treat $\mathrm{H} 9 \mathrm{c} 2$ cells.

Cell transfection. ENPP2 and FoxO4 were overexpressed in $\mathrm{H} 9 \mathrm{c} 2$ cells to produce overexpressing (Oe)-ENPP2 and Oe-FoxO4 cell lines. The rat ENPP2 and FoxO4 cDNA sequences were synthesized by GenScript and inserted into the pcDNA3.1 vector (Invitrogen; Thermo Fisher Scientific, Inc.). An empty pcDNA3.1 vector was used as the negative control (NC). H9c2 cells were seeded into 6-well plates at the density of $4 \times 10^{5}$ cells/well $10 \mathrm{~h}$ prior to transfection, and the vectors $(2.5 \mu \mathrm{g})$ were transfected into cells using Lipofectamine ${ }^{\circledR} 2000$ reagent (Invitrogen; Thermo Fisher Scientific, Inc.) according to the manufacturer's instructions, at $37^{\circ} \mathrm{C}$ for $48 \mathrm{~h}$. At $48 \mathrm{~h}$ post-transfection, the cells were exposed to $5 \mu \mathrm{M}$ DOX for $24 \mathrm{~h}$ at $37^{\circ} \mathrm{C}$ and collected for subsequent analysis. Control cells were devoid of treatment.

Cell viability assessment. Cell viability was determined using the MTT assay kit (Beyotime Institute of Biotechnology). Briefly, control or transfected $\mathrm{H} 9 \mathrm{c} 2$ cells were seeded into 96-well plates at the density of $2 \times 10^{3}$ cells/well. Following treatment with DOX for 12,24 or $48 \mathrm{~h}$, the culture medium was replaced with DMEM without DOX and then added to $10 \mu \mathrm{l} \mathrm{MTT}$ solution at $37^{\circ} \mathrm{C}$ for $4 \mathrm{~h}$. The cells were incubated with formazan lysis solution at room temperature for 10 min until the purple crystals were completely dissolved. Finally, the absorbance was measured at $570 \mathrm{~nm}$ using a spectrophotometer.
Oxidative stress measurement. To detect cellular total ROS status, the $2 \times 10^{3}$ cells/well were seeded into 96 -well plates and stained with $2^{\prime}, 7^{\prime}$-dichlorodihydrofluorescein diacetate (Abcam) at $37^{\circ} \mathrm{C}$ for $45 \mathrm{~min}$. Fluorescence was measured at excitation and emission wavelengths of 485 and $535 \mathrm{~nm}$ using a multimode plate reader (PerkinElmer, Inc.).

The increase in ROS levels was also examined using a commercially available thiobarbituric acid reactive substances (TBARS) assay kit (cat. no. 700870; Cayman Chemical Company) according to the manufacturer's instructions. GSH peroxidase (GSH-Px) activity was measured using a GSH-PX assay kit (cat. no. A005-1-2; Nanjing Jiancheng Bioengineering Institute) in accordance with the manufacturer's instructions.

Measurement of $\mathrm{Fe}^{2+}$ activity. Cellular $\mathrm{Fe}^{2+}$ activity was measured using the Iron Assay kit (Abcam) according to the manufacturer's instructions. Briefly, control or transfected $\mathrm{H} 9 \mathrm{c} 2$ cells were seeded into 96-well plates at the density of $1 \times 10^{3}$ cells/well. Following treatment with $5 \mu \mathrm{M}$ DOX for $24 \mathrm{~h}$ at $37^{\circ} \mathrm{C}, 5 \mu \mathrm{l}$ assay buffer was added to each sample and incubated at $37^{\circ} \mathrm{C}$ for $30 \mathrm{~min}$. Following addition of $100 \mu \mathrm{l}$ Iron Probe to each well and incubation at $37^{\circ} \mathrm{C}$ for $60 \mathrm{~min}$ in the dark, the optical density $(593 \mathrm{~nm})$ was measured using a colorimetric microplate reader.

Dual-luciferase reporter assay. The JASPAR database (http://jaspar.genereg.net/) was searched and FoxO4 was identified as a potential target that could bind to the promoter of ENPP2. To confirm the interaction between FoxO4 and the ENPP2 promoter, a dual-luciferase reporter assay was performed. Wild-type or mutant ENPP2 E1 and E2 sequences were amplified and cloned downstream of the luciferase reporter gene in pMIR-REPORT luciferase vectors (Thermo Fisher Scientific, Inc.). Subsequently, they were co-transfected with pcDNA3.1-FoxO4 (Oe-FoxO4; $2.5 \mu \mathrm{g}$ ) or Oe-NC $(2.5 \mu \mathrm{g})$ using Lipofectamine 2000 reagent. At $48 \mathrm{~h}$ post-transfection, luciferase activity was measured with a dual-luciferase reporter system (Promega Corporation). Renilla luciferase activity was used for normalization.

Chromatin immunoprecipitation (ChIP) assay. H9c2 cells were crosslinked with $1 \%$ formaldehyde for $10 \mathrm{~min}$ at $37^{\circ} \mathrm{C}$ and a ChIP assay was performed with a high-sensitivity kit (Abcam). Cells were washed with cold PBS and suspended in $1 \mathrm{ml}$ PBS containing $5 \mu \mathrm{l}$ protease inhibitors. Cells were centrifuged at $4^{\circ} \mathrm{C}$ at $716 \times \mathrm{g}$ for $5 \mathrm{~min}$. A total of $300 \mu \mathrm{l} \mathrm{SDS} \mathrm{lysis}$ buffer (1\% SDS, $10 \mathrm{mM}$ EDTA and $50 \mathrm{mM}$ Tris- $\mathrm{HCl} \mathrm{pH} 8.0$ ) was then used to lyse the cells, which were subsequently sonicated at $150 \mathrm{~Hz}$ and sheared with four sets of $10 \mathrm{sec}$ pulses on wet ice using a high intensity ultrasonic processor. An equal amount of chromatin $(100 \mu \mathrm{l})$ was immunoprecipitated at $4^{\circ} \mathrm{C}$ overnight. The antibodies used in this assay included anti-FoxO4 (cat. no. ab128908; 1:50; Abcam) and IgG (as the NC; cat. no. ab172730; 1:50; Abcam). Immunoprecipitated products were collected after incubation with magnetic beads coupled with anti-IgG or anti-FoxO4. The beads were washed using a magnetic separation rack and the bound chromatin was eluted in ChIP Elution Buffer with Proteinase K mixer. The primer sequences used for ENPP2 detection were as follows: Forward 5'-TTCCAATGTACCCCGCCTTC-3' and reverse 
5'-AGCTGCCTTCCACATACTGTT-3'. The recovered DNA fragments were evaluated via RT-qPCR. The relative level of ENPP2 was normalized according to the average level of the IgG group.

Lipid ROS measurement. Lipid ROS generation was measured by adding C11-BODIPY (Invitrogen; Thermo Fisher Scientific, Inc.) at a final concentration of $1.5 \mu \mathrm{M}$ for $20 \mathrm{~min}$ at $37^{\circ} \mathrm{C}$ before cell harvesting, according to the manufacturer's instructions. Lipid ROS-positive cells were finally assessed using a FACSCanto II flow cytometer (BD Biosciences). The lipid ROS can be differentiated by adding C11-BODIPY, which is a lipid soluble, ratio type fluorescent probe used to indicate lipid peroxidation and antioxidant properties in model membrane systems and in living cells (14).

Reverse transcription-quantitative PCR (RT-qPCR). Total RNA was isolated from $\mathrm{H} 9 \mathrm{c} 2$ cells using TRIzol $^{\circledR}$ reagent (Invitrogen; Thermo Fisher Scientific, Inc.). The sample concentration was quantified via spectrophotometry. Total RNA was reverse transcribed into cDNA using the PrimeScript RT reagent kit (Takara Bio, Inc.) according to the manufacturer's instructions. qPCR was performed using a SYBR ${ }^{\circledast}$ Premix Ex Taq kit (Takara Bio, Inc.) in accordance with the manufacturer's instructions. The thermocycling conditions were as follows: Initial denaturation for $95^{\circ} \mathrm{C}$ for $5 \mathrm{~min}$, followed by 40 cycles of denaturation at $95^{\circ} \mathrm{C}$ for $15 \mathrm{sec}$ and annealing at $60^{\circ} \mathrm{C}$ for $30 \mathrm{sec}$; and a final extension of $10 \mathrm{~min}$ at $72^{\circ} \mathrm{C}$. The fold difference in gene expression was calculated using the $2^{-\Delta \Delta \mathrm{Cq}}$ method (15) following normalization to the expression levels of GAPDH mRNA. The primer sequences were as follows: ENPP2 forward, 5'-TTCCAATGT ACCCCGCCTTC-3' and reverse, 5'-AGCTGCCTTCCACAT ACTGTT-3'; FoxO4 forward, 5'-AGCGACTGACACTTGCCC AGAT-3' and reverse, 5'- AGGGTTCAGCATCCACCAAGA G-3'; and GAPDH forward, 5'-CGTGCCGCCTGGAGAA-3' and reverse, 5'-CCCTCAGATGCCTGCTTCAC-3'.

Western blot analysis. Total proteins were isolated from H9c2 cells using RIPA lysis buffer (Beyotime Institute of Biotechnology). Protein concentration was determined using a BCA protein assay kit (Beyotime Institute of Biotechnology) and proteins ( $40 \mu \mathrm{g} / \mathrm{lane}$ ) were separated via $12 \%$ SDS-PAGE and subsequently transferred to a PVDF membrane. The membranes were blocked with $10 \%$ non-fat dry milk at room temperature for $2 \mathrm{~h}$ and incubated with primary antibodies at $4^{\circ} \mathrm{C}$ overnight. Following the primary antibody incubation, the membranes were incubated with HRP-conjugated anti-rabbit or anti-mouse IgG secondary antibodies. The immunoreactive bands were visualized using DAB (Wuhan Boster Biological Technology, Ltd.). GAPDH was used as a loading control, which was in accordance with the previous studies showing that GAPDH expression could be used as a loading control in for DOX investigation (16-18). Densitometric analysis was performed using ImageJ software (version 1.52r; National Institutes of Health). Anti-LC3I/II (cat. no. 4108S; 1:1,000), anti-Beclin1 (cat. no. 3495T; 1:1,000), anti-autophagy related 5 (ATG5; cat. no. 12994T; 1:1,000), anti-p62 (cat. no. 23214S; 1:1,000) and anti-GAPDH (cat. no. 5174T; 1:1,000) antibodies were purchased from Cell Signaling Technology, Inc. Anti-FoxO4 (cat. no. ab128908; 1:1,000), anti-ENPP2 (cat. no. ab140915; 1:1,000), anti-solute carrier family 7 member 11 (SLC7A11; cat. no. ab175186; 1:1,000), anti-GPX4 (cat. no. ab125066; 1:1,000), anti-ferroportin 1 (FPN1; cat. no. ab239511; 1:1,000), anti-NADPH oxidase 4 (NOX4; cat. no. ab133303; 1:1,000), HRP-conjugated anti-mouse (cat. no. ab6728; 1:5,000) and HRP-conjugated anti-rabbit IgG (cat. no. ab6721; 1:5,000) antibodies were provided by Abcam.

Statistical analysis. All experiments were repeated three times and the results are presented as the mean \pm SD. Data were analyzed using GraphPad Prism 8 software (GraphPad Software, Inc.). Comparisons between two groups were conducted using an unpaired Student's t-test, while comparisons among multiple groups were performed using one-way ANOVA followed by Tukey's post hoc test. $\mathrm{P}<0.05$ was considered to indicate a statistically significant difference.

\section{Results}

ENPP2 expression is downregulated following DOX treatment. Different concentrations $(0.625,1.25,2.5,5$ and $10 \mu \mathrm{M})$ of DOX were used to treat $\mathrm{H} 9 \mathrm{c} 2$ cells for 12,24 and $48 \mathrm{~h}$, respectively. Cell viability was significantly reduced following DOX treatment in a time- and dose-dependent manner (Fig. 1A). In addition, the protein expression level of ENPP2 were significantly decreased compared with those of the control cells, following treatment of the cells with 2.5, 5 and $10 \mu \mathrm{M}$ DOX for $24 \mathrm{~h}$ and treatment of the cells with $5 \mu \mathrm{M}$ DOX for 24 and $48 \mathrm{~h}$. In addition, the mRNA expression levels of ENPP2 were downregulated in cells following DOX treatment in a dose- and time-dependent manner (Fig. 1B-E). Moreover, $5 \mu \mathrm{M}$ DOX treatment was used for $24 \mathrm{~h}$ to treat H9c2 cells in subsequent experiments.

Overexpression of ENPP2 enhances autophagy and inhibits ferroptosis induced by DOX in H9c2 cardiomyocytes. Overexpression of ENPP2 was achieved in H9c2 cells and the results verified the effective increase of ENPP2 protein and mRNA expression levels (Fig. 2A and B). Subsequently, control or ENPP2-Oe cells were exposed to DOX in order to examine the changes noted in cell viability, oxidative stress and autophagy. The reduction in cell viability caused by DOX induction was effectively rescued by ENPP2 overexpression (Fig. 2C). As shown in Fig. 2D, DOX induction significantly elevated in the level of total ROS compared with the control group, which was notably restored by ENPP2 overexpression. Moreover, DOX treatment resulted in a significant increase in TBARS content and a decrease in GSH-Px expression, which were both partially recovered by ENPP2 overexpression (Fig. 2E and F).

The expression levels of the proteins involved in autophagy, including LC3II/I, Beclin 1, ATG5 and p62, were also assessed. The protein expression levels of LC3II/I, Beclin 1 and ATG5 were significantly increased, but p62 expression was decreased following DOX treatment, indicating the induction of autophagy by DOX in H9c2 cells (Fig. 2G). Concomitantly, ENPP2 overexpression enhanced the changes noted in these proteins following induction of autophagy by DOX.

To evaluate the induction of ferroptosis in DOX-treated $\mathrm{H} 9 \mathrm{c} 2$ cells, $\mathrm{Fe}^{2+}$ activity, lipid ROS production and the 


\section{A}

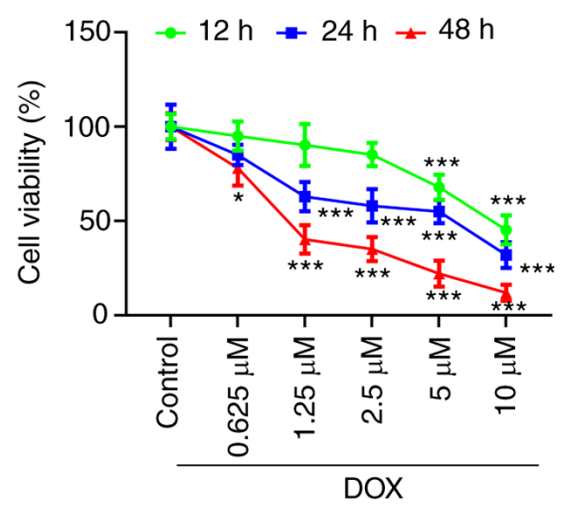

B
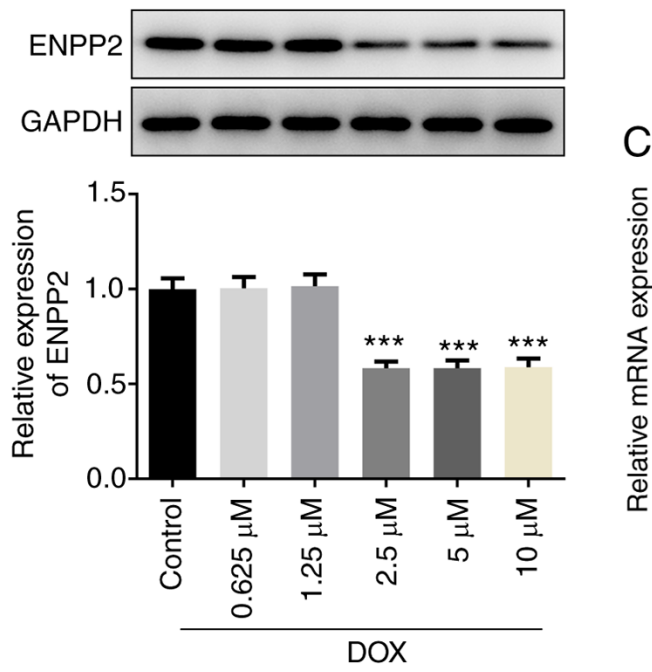

$\mathrm{C}$

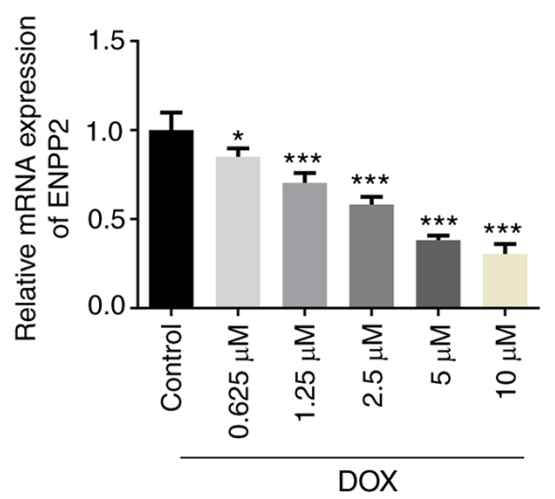

D
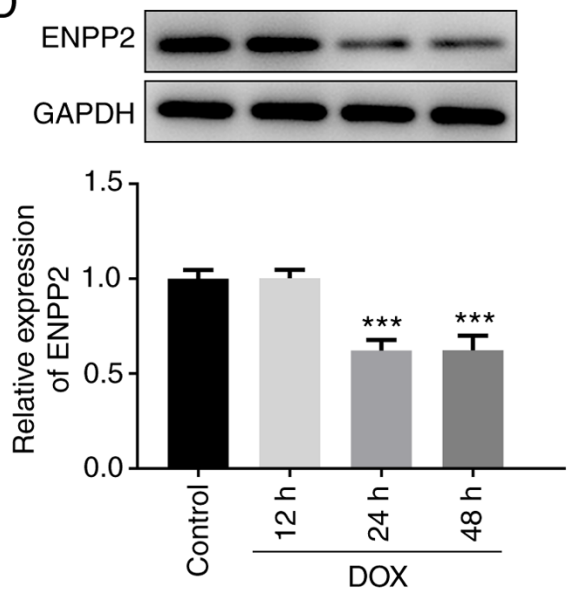

E

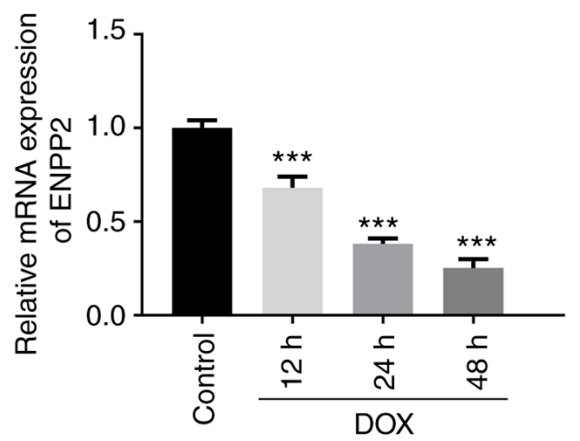

Figure 1. Effects of DOX on ENPP2 expression in H9c2 cardiomyocytes. (A) H9c2 cells were exposed to different concentrations of DOX for 12, 24 and 48 h, and cell viability was measured using the MTT assay. H9c2 cells were exposed to different concentrations of DOX for $24 \mathrm{~h}$ and subsequently the ENPP2 (B) protein and (C) mRNA expressions levels were detected. H9c2 cells were exposed to $5 \mu \mathrm{M}$ DOX for 12,24 and 48 h, and the ENPP2 (D) protein and (E) mRNA expression levels were detected. ${ }^{*} \mathrm{P}<0.05$ and ${ }^{* * *} \mathrm{P}<0.001$ vs. control. DOX, doxorubicin; ENPP2, ectonucleotide pyrophosphatase/phosphodiesterase 2 .

expression levels of ferroptosis-associated proteins, including SLC7A11, GPX4, FPN1 and NOX4, were determined. DOX treatment resulted in a significant increase in cellular $\mathrm{Fe}^{2+}$ activity and lipid ROS production (Fig. 3A and B), while the cells that overexpressed ENPP2 exhibited decreased $\mathrm{Fe}^{2+}$ activity and lipid ROS production compared with the cells transfected with NC vectors (Fig. 3A and B). Furthermore, the decreased expression levels of SLC7A11, GPX4 and FPN1 and increased expression level of NOX4 caused by DOX were significantly reversed by the overexpression of ENPP2 (Fig. 3C). These data suggested the inhibitory effects of ENPP2 on DOX-induced ferroptosis in H9c2 cells.

FoxO4 binds to the promoter of ENNP2 and regulates ENPP2 expression in H9c2 cardiomyocytes. FoxO4 was predicted to bind to the E1 and E2 sequences of the ENPP2 promoter (Fig. 4A). As displayed in Fig. 4B, the protein and mRNA expression levels of FoxO4 were significantly upregulated after transfection with FoxO4 plasmid. Additionally, FoxO4 overexpression significantly reduced ENPP2 mRNA expression, suggesting that FoxO4 may downregulate ENPP2 expression by binding to the ENPP2 promoter (Fig. 4C). Then, the expression level of ENPP2 in DOX-induced H9c2 cells with FoxO4 overexpression was assessed using RT-qPCR. It was found that gain-function of FoxO4 significantly downregulated the expression level of ENPP2 compared with the DOX + Oe-NC group (Fig. 4D). Subsequently, the interaction between FoxO4 and ENPP2 was validated using dual-luciferase reporter (Fig. 4E) and ChIP (Fig. 4F) assays. These results suggested that FoxO4 could bind to the promoter of ENNP2 and regulate ENPP2 expression in H9c2 cardiomyocytes.

Overexpression of FoxO4 partially reverses the effects of ENPP2 on DOX-induced autophagy and ferroptosis in H9c2 cardiomyocytes. Finally, FoxO4 and ENPP2 were co-overexpressed in H9c2 cells, which had been treated with DOX. Cell viability was reduced in cells that co-overexpressed FoxO4 and ENPP2 compared with cells that only overexpressed ENPP2 (Fig. 5A). This indicated that FoxO4 overexpression inhibited the protective effect of ENPP2 on cell viability, which was impaired by DOX (Fig. 5A). Moreover, the effects 


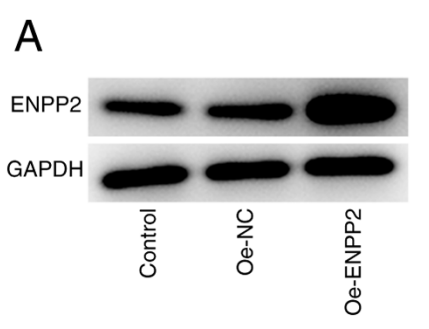

D

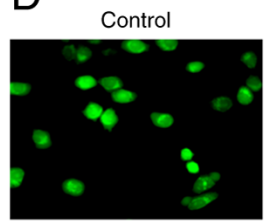

$\mathrm{DOX}+\mathrm{Oe}-\mathrm{NC}$

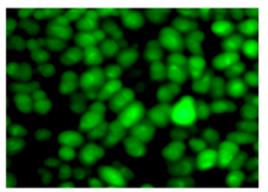

G

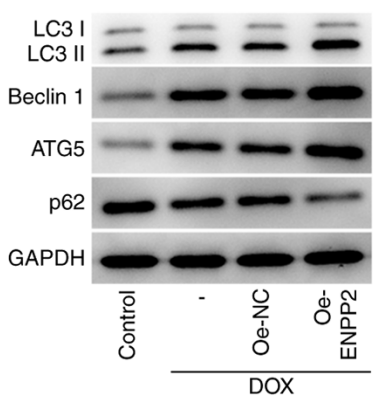

DOX

DOX+Oe-ENPP2
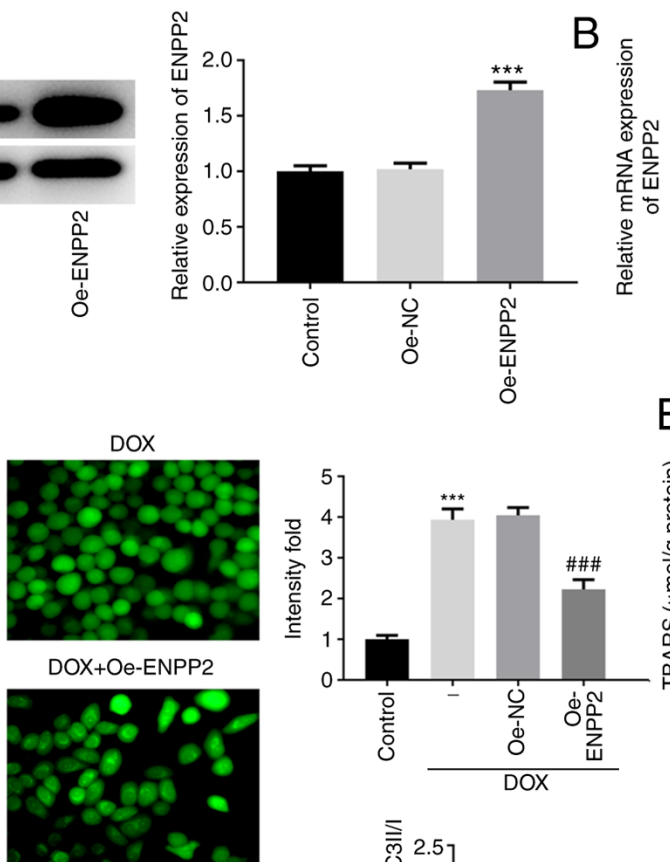

$\mathrm{E}$
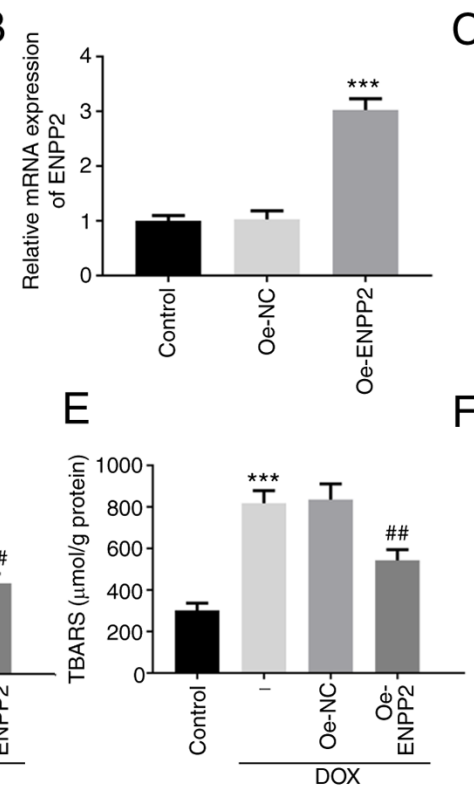

F
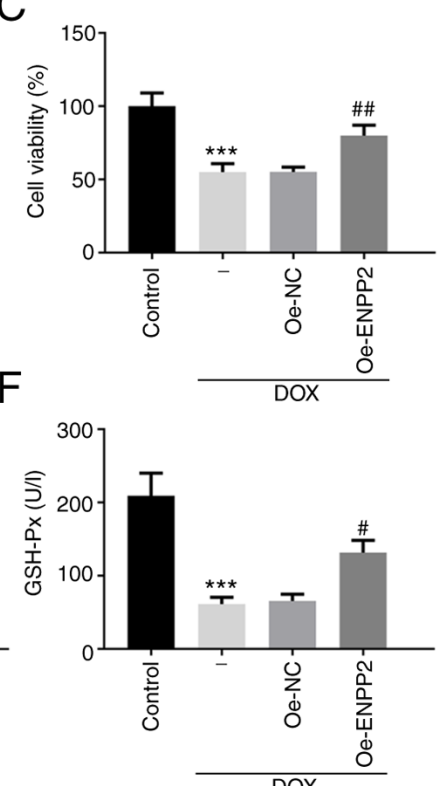
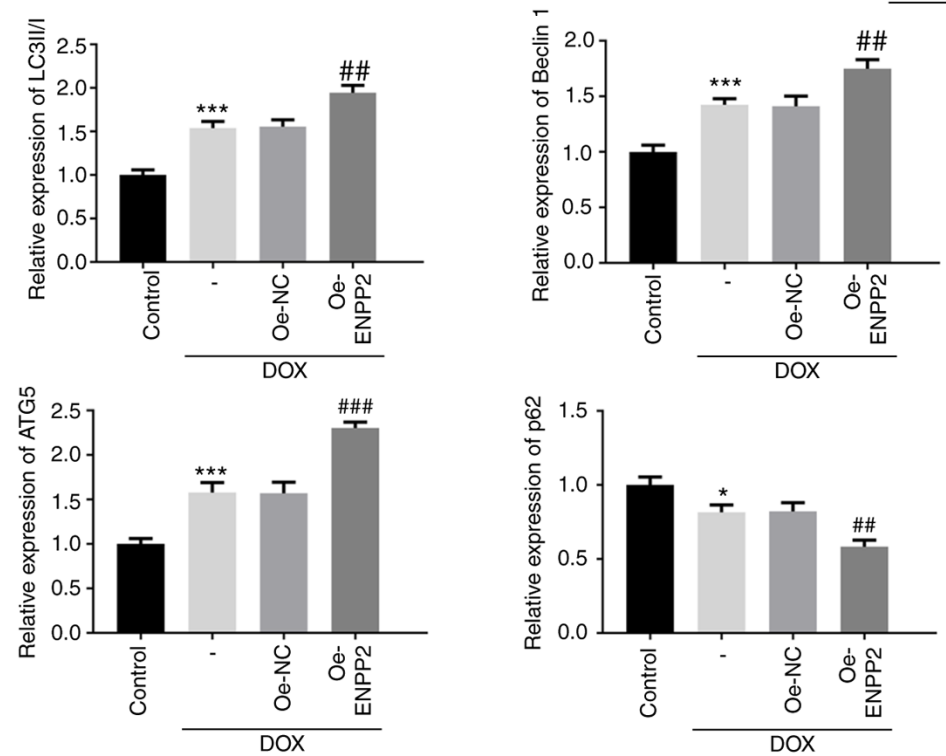

Figure 2. Effects of ENPP2 overexpression on DOX-induced autophagy in H9c2 cardiomyocytes. H9c2 cells were transfected with ENPP2 vector in order to obtain ENPP2-Oe cells. The transfection efficiency was detected via (A) western blotting and (B) reverse transcription-quantitative PCR. ${ }^{* * * *} \mathrm{P}<0.001$ vs. Oe-NC. (C) ENPP2-Oe H9c2 cells were exposed to $5 \mu \mathrm{M}$ DOX for $24 \mathrm{~h}$ and cell viability was measured. (D) The total reactive oxygen species production was assessed using a 2',7'-dichlorodihydrofluorescein diacetate kit (magnification, $\mathrm{x} 200$ ). ENPP2-Oe H9c2 cells were exposed to $5 \mu \mathrm{M}$ DOX for $24 \mathrm{~h}$, and subsequently (E) TBARS concentration levels and (F) GSH-Px expression levels were measured. (G) ENPP2-Oe H9c2 cells were exposed to $5 \mu$ M DOX for $24 \mathrm{~h}$ and the expression levels of the proteins involved in autophagy were evaluated. ${ }^{*} \mathrm{P}<0.05$ and ${ }^{* * *} \mathrm{P}<0.001$ vs. control; ${ }^{*} \mathrm{P}<0.05$, ${ }^{\# \#} \mathrm{P}<0.01$ and ${ }^{\# \# \#} \mathrm{P}<0.001$ vs. Oe-NC. DOX, doxorubicin; NC, negative control; TBARS, thiobarbituric acid reactive substances; GSH-Px, glutathione peroxidase; ENPP2, ectonucleotide pyrophosphatase/phosphodiesterase 2; Oe, overexpressing; ATG5, autophagy related 5.

of ENPP2 overexpression on the total ROS levels, TBARS concentration and GSH-Px expression were partially reversed by FoxO4 overexpression (Fig. 5B-E). Similar results were observed in Fig. 5F, as the co-transfection with FoxO4 and ENPP2 plasmids significantly decreased the levels of LC3II/I, Beclin 1 and ATG5 and increased the expression of p62 compared with those in the DOX + Oe-ENPP2 group. In addition, the inhibitory effects of ENPP2 on DOX-induced ferroptosis were significantly reduced by FoxO4 overexpression, as demonstrated by increased $\mathrm{Fe}^{2+}$ and lipid ROS activity levels, decreased SLC7A11, GPX4 and FPN1 expression, and increased NOX4 expression, which were observed following FoxO4 overexpression (Fig. 6A-C).

\section{Discussion}

DOX is a potent anticancer drug clinically applied for the treatment of various cancer types, such as breast, colon and bladder cancer (19-21). However, its cardiotoxicity is a major challenge, thereby limiting its clinical use. Therefore, further understanding of the molecular mechanisms involved in DOX function can alleviate cardiotoxicity and improve the clinical efficacy of this chemotherapeutic drug. Currently, no effective method exists to relieve the cardiac dysfunction caused by DOX. Thus, the development of effective treatments is a major focus for improving the side effects of DOX. The results of the present study found that ENPP 2 mRNA 

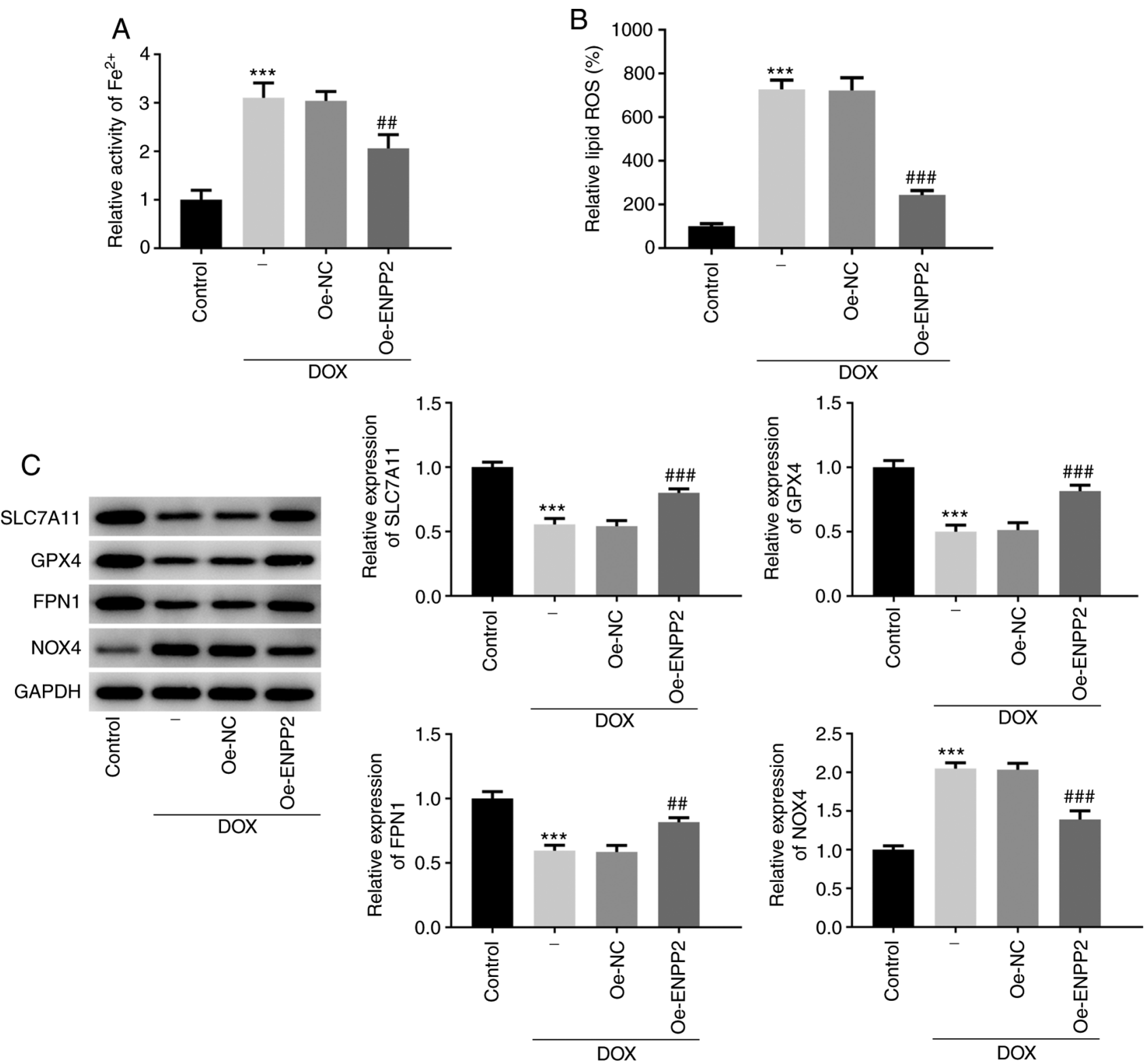

Figure 3. Effects of ENPP2 overexpression on DOX-induced ferroptosis in H9c2 cardiomyocytes. (A) H9c2 cells that overexpressed ENPP2 were exposed to $5 \mu \mathrm{M}$ DOX for $24 \mathrm{~h}$ and cellular $\mathrm{Fe}^{2+}$ activity was measured using an Iron Assay kit. (B) The lipid ROS levels were assessed. (C) The expression levels of the proteins involved in ferroptosis were measured using western blot analysis. ${ }^{* * *} \mathrm{P}<0.001$ vs. control; ${ }^{\# \#} \mathrm{P}<0.01$ and ${ }^{\# \# \#} \mathrm{P}<0.001$ vs. Oe-NC. DOX, doxorubicin; ROS, reactive oxygen species; DCFDA, 2',7'-dichlorodihydrofluorescein diacetate; NC, negative control; ENPP2, ectonucleotide pyrophosphatase/phosphodiesterase 2; Oe, overexpressing; SLC7A11, solute carrier family 7 member 11; GPX4, GSH peroxidase 4; FPN1, ferroportin 1; NOX4, NADPH oxidase 4.

and protein expression levels were downregulated following DOX treatment. Gene expression can be divided into two levels: Transcription and translation, that is, the mRNA level and the protein level. The time and site of transcription and translation of gene expression are spatiotemporal. After transcription, there will be post-transcriptional processing, degradation of transcriptional products, translation, post-translation processing and modification. Therefore, transcription levels and translation levels are not completely consistent, which may explain the different degrees of decline between the mRNA and protein expression levels of ENPP2 (22). In the present study, FoxO4 was found to bind to the ENNP2 promoter to improve DOX-induced cardiotoxicity via the induction of ferroptosis.
Oxidative stress and dysregulation of autophagy have been mainly reported to participate in the cardiotoxicity of DOX $(23,24)$. In the present study, the results demonstrated that DOX treatment impaired cell viability, increased TBARS concentration levels and reduced GSH-Px and p62 expression in $\mathrm{H} 9 \mathrm{c} 2$ cardiomyocytes. Concomitantly, DOX increased LC3II/I, Beclin 1 and ATG5 expression levels. The results of the present study confirmed that DOX induced oxidative stress and autophagy in cardiomyocytes. In addition, the relative $\mathrm{Fe}^{2+}$ activity and lipid ROS production were enhanced, whereas the expression levels of SLC7A11, GPX4 and FPN1 were decreased following treatment of the cells with DOX. In contrast to these findings, NOX4 expression was increased following incubation of the cells with DOX. Thus, ROS accumulation 
A
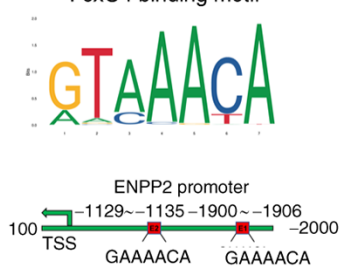

D

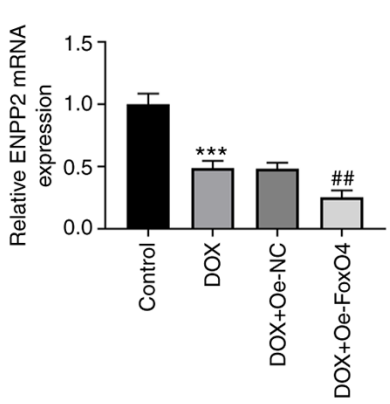

B

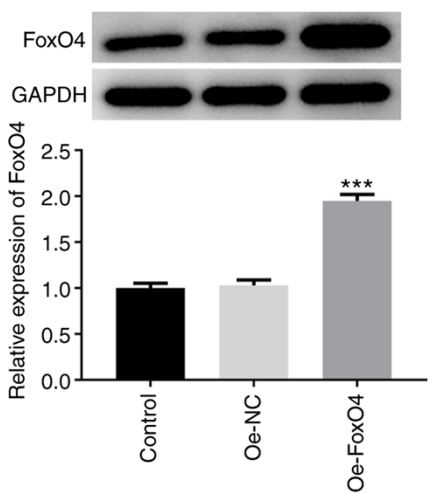

E

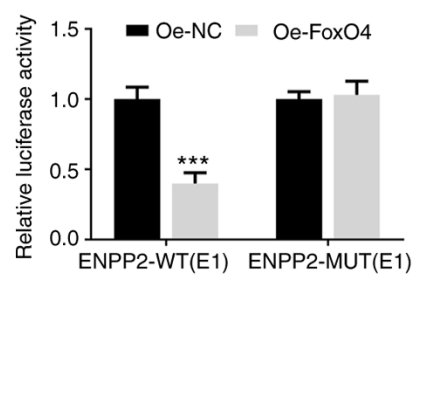

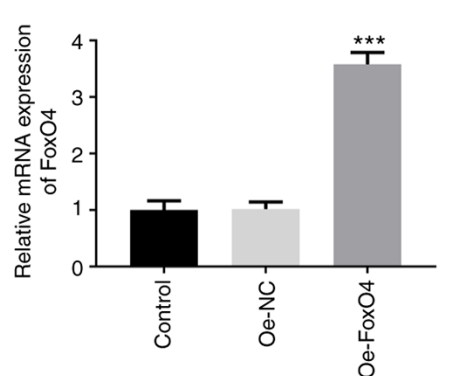

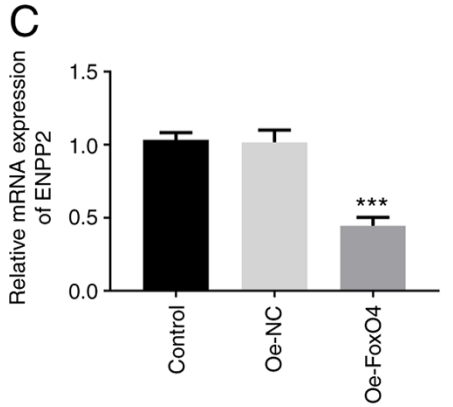

F
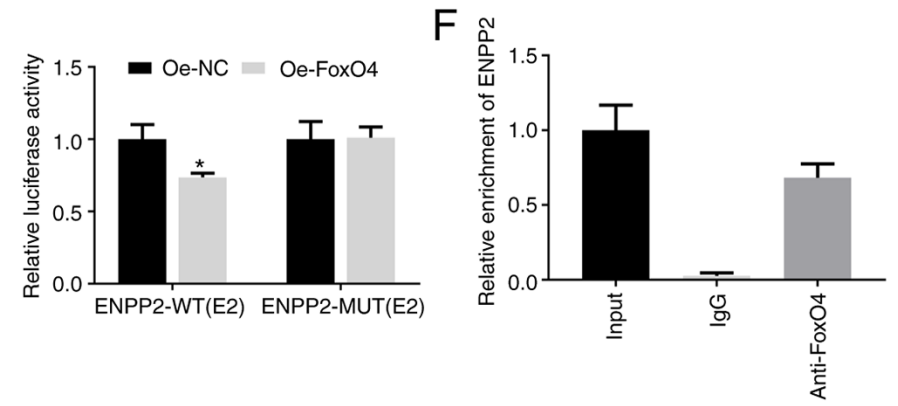

Figure 4. Association between ENPP2 and FoxO4 in H9c2 cardiomyocytes. (A) The binding sequences of FoxO4 and the ENPP2 promoter. (B) FoxO4-Oe $\mathrm{H} 9 \mathrm{c} 2$ cells were established and the transfection efficiency was assessed via western blot analysis and RT-qPCR. ${ }^{* * *} \mathrm{P}<0.001$ vs. Oe-NC. (C) mRNA expression levels of ENPP2 in H9c2 cells with or without FoxO4 overexpression were measured. ${ }^{* * *} \mathrm{P}<0.001$ vs. Oe-NC. (D) The expression level of ENPP2 in DOX-induced H9c2 cells with FoxO4 overexpression was assessed using RT-qPCR. ${ }^{* * *} \mathrm{P}<0.001$ vs. control; ${ }^{\#} \mathrm{P}<0.01$ vs. DOX + Oe-NC. The interaction between FoxO4 and the ENPP2 promoter was confirmed by (E) dual-luciferase reporter and the (F) chromatin immunoprecipitation assays. * $<0.05$ and ${ }^{* * *} \mathrm{P}<0.001$ vs. Oe-NC. NC, negative control; ENPP2, ectonucleotide pyrophosphatase/phosphodiesterase 2; Oe, overexpressing; DOX, doxorubicin; RT-qPCR, reverse transcription-quantitative PCR.

in DOX-treated cardiomyocytes was accompanied with deregulation of their antioxidant defense system. GSH serves a major role in the cellular antioxidant defense (25). Depletion of GSH leads to accumulation of lipid ROS levels, protein or membrane damage and subsequent ferroptotic cell death (26). Cellular iron is required in normal life processes, such as DNA synthesis, metastasis, cell cycle progression, angiogenesis and/or mitochondrial iron metabolism. Ferritin is the iron storage protein of the cells. $\mathrm{Fe}^{2+}$ promotes lipid peroxide accumulation via the Fenton reaction and lipid oxidation (27). GPX4 can prevent the toxicity of lipid peroxides by its enzyme activity. It can also maintain the homeostasis of membrane lipid bilayers. Furthermore, GSH is the co-factor of GPX4 and aids the catalysis of peroxides into alcohols. Downregulation of GSH expression directly inactivates GPX4 and leads to subsequent induction of ferroptosis (28). Therefore, the present results, when taken together, demonstrated that DOX could induce ferroptosis.

Ferroptosis was recently reported as a novel mechanism of iron-dependent regulated cell death (27). It has been proposed that ferroptosis serves a significant role in DOX-induced cardiotoxicity, and ferroptosis inhibition protects against this process $(4,5)$. Moreover, ENPP2 has been shown to inhibit ferroptosis in cardiomyocytes (10). In the present study, DOX treatment caused the downregulation of ENPP2 expression. It was hypothesized that ENPP2 may protect cardiomyocytes from DOX-induced cardiotoxicity by inhibiting ferroptosis. Therefore, this enzyme was overexpressed in H9c2 cells, which were subsequently treated with DOX. Numerous regulators, such as GPX4, SLC7A11, FPN1 and NOX4, have been identified to be involved in regulation of cell ferroptosis (29-31). It has been reported that ENPP2 overexpression enhances the expression levels of the ferroptosis-associated gene GPX4 in H9c2 cells (10). The current results indicated that ENPP2 overexpression partially recovered TBARS, GSH-Px and ROS levels, which in turn enhanced autophagy and inhibited ferroptosis. Autophagy exerts a dual function in the regulation and progression of cardiac dysfunction. The induction of autophagy has been shown to attenuate DOX-induced cardiotoxicity. In addition, autophagy has been reported to regulate ferroptosis (32-34). In the present study, ENPP2 inhibited ferroptosis by blocking the oxidative stress response, which in turn reduced ROS production and enhanced the induction of autophagy. These processes then promoted the elimination of long-lived proteins and damaged organelles.

The JASPAR database was searched and FoxO4 was identified as a potential target that could bind to the promoter of ENPP2. Additional analysis validated the direct interaction between FoxO4 and ENPP2. It was found that FoxO4 overexpression reduced ENPP2 expression. FoxO4 regulates negatively gene transcription via post-transcriptional suppression of coding mRNAs (35). The results of the present study indicated that FoxO4 may negatively control ENPP2 transcription by binding to the ENPP2 promoter. Notably, FoxO4 has been extensively investigated with regards to its effects on promoting myocardial injury, and this transcription factor 
A

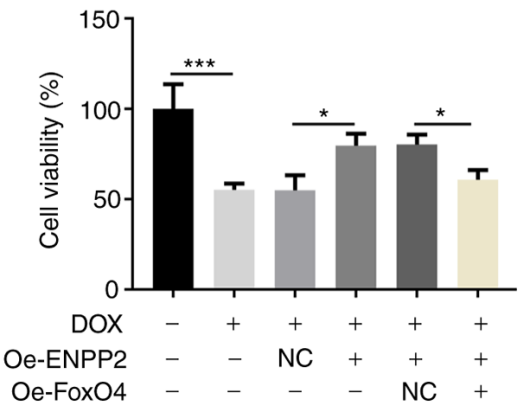

$\mathrm{B}$
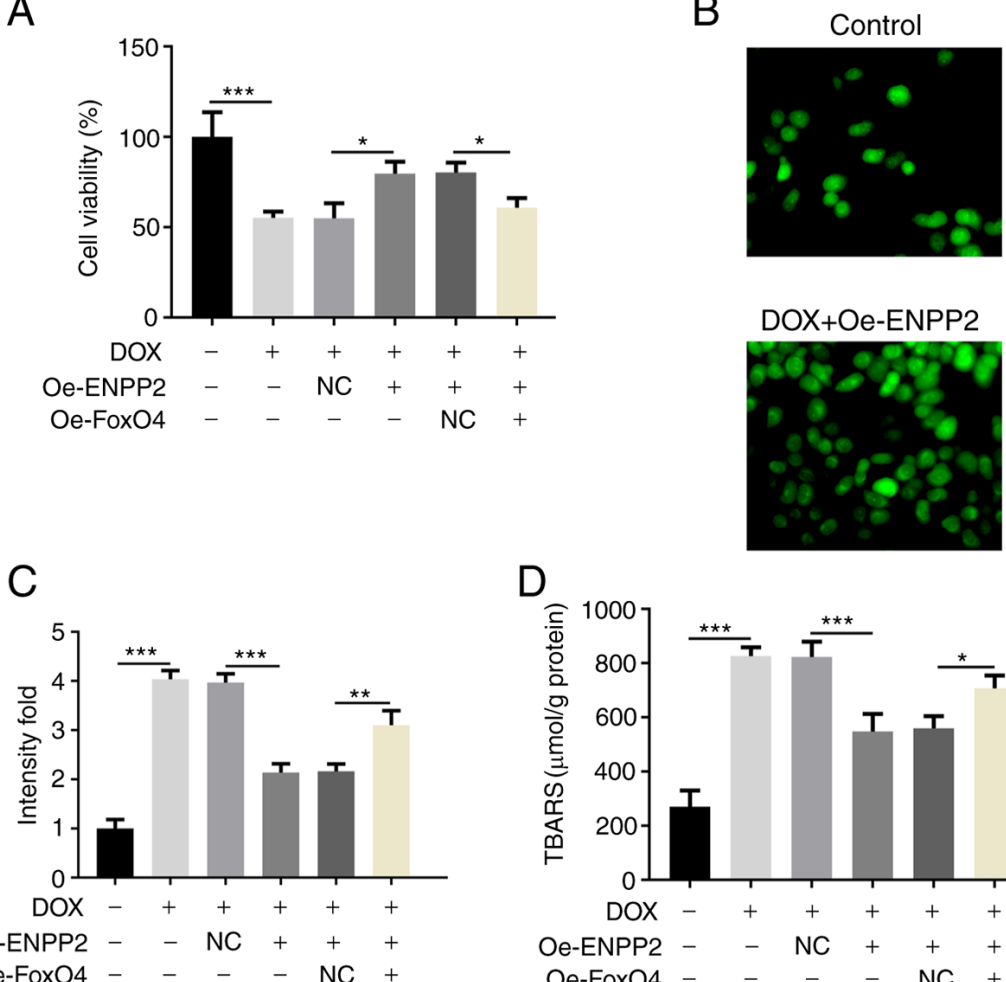

$\mathrm{F}$

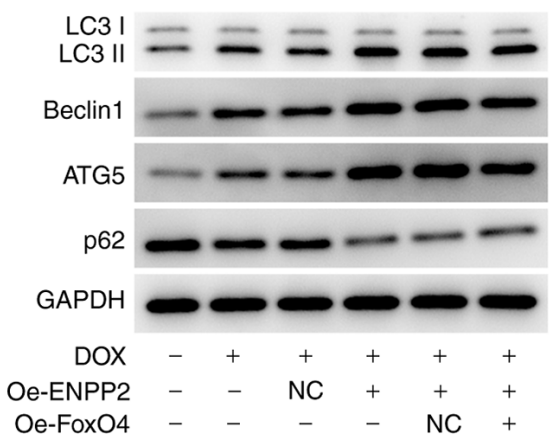

$\mathrm{D}$
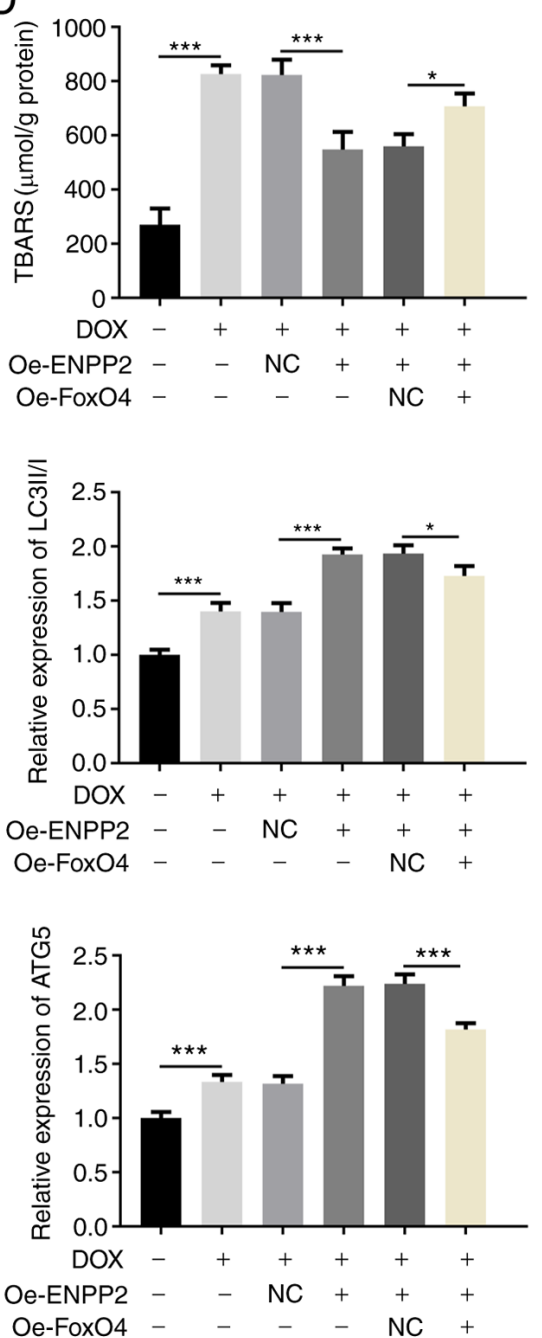

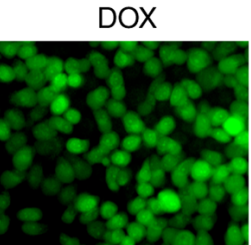

DOX+Oe-ENPP2

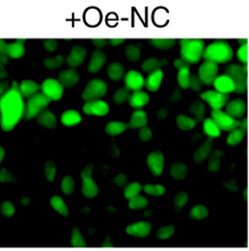

E
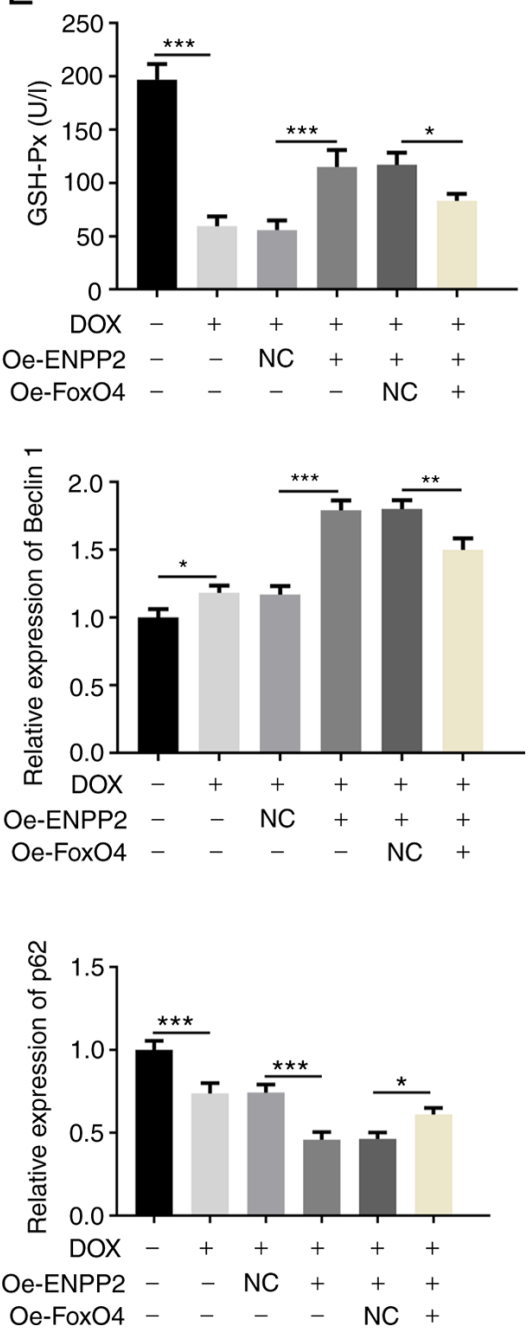

Figure 5. FoxO4 overexpression inhibits the effects of ENPP2 overexpression on DOX-induced autophagy in H9c2 cardiomyocytes. H9c2 cells that co-overexpressed FoxO4 and ENPP2 were exposed to $5 \mu \mathrm{M}$ DOX for $24 \mathrm{~h}$. Subsequently, (A) cell viability was measured with a MTT assay. (B) Total reactive oxygen species production was assessed using the DCFDA kit (magnification, x200), (C) and the results were quantified. (D) TBARS concentration levels and (E) GSH-Px expression levels were detected using their corresponding kits. (F) The expression levels of the proteins involved in autophagy were evaluated via western blot analysis. ${ }^{*} \mathrm{P}<0.05,{ }^{* *} \mathrm{P}<0.01$ and ${ }^{* * * *} \mathrm{P}<0.001$. DOX, doxorubicin; TBARS, thiobarbituric acid reactive substances; GSH-Px, glutathione peroxidase; ENPP2, ectonucleotide pyrophosphatase/phosphodiesterase 2; Oe, overexpressing; NC, negative control; ATG5, autophagy related 5.

is involved in myocardial ischemia/reperfusion, myocardial infarction and DOX-induced cardiotoxicity $(12,13,36)$. Subsequently, the overexpression of FoxO4 in ENPP2-Oe H9c2 cells was assessed with regards to the effects of ENPP2 on DOX-induced cardiotoxicity. FoxO4 overexpression inhibited all the effects caused by ENPP2 overexpression on DOX-induced cardiotoxicity, which supported the initial hypothesis. 

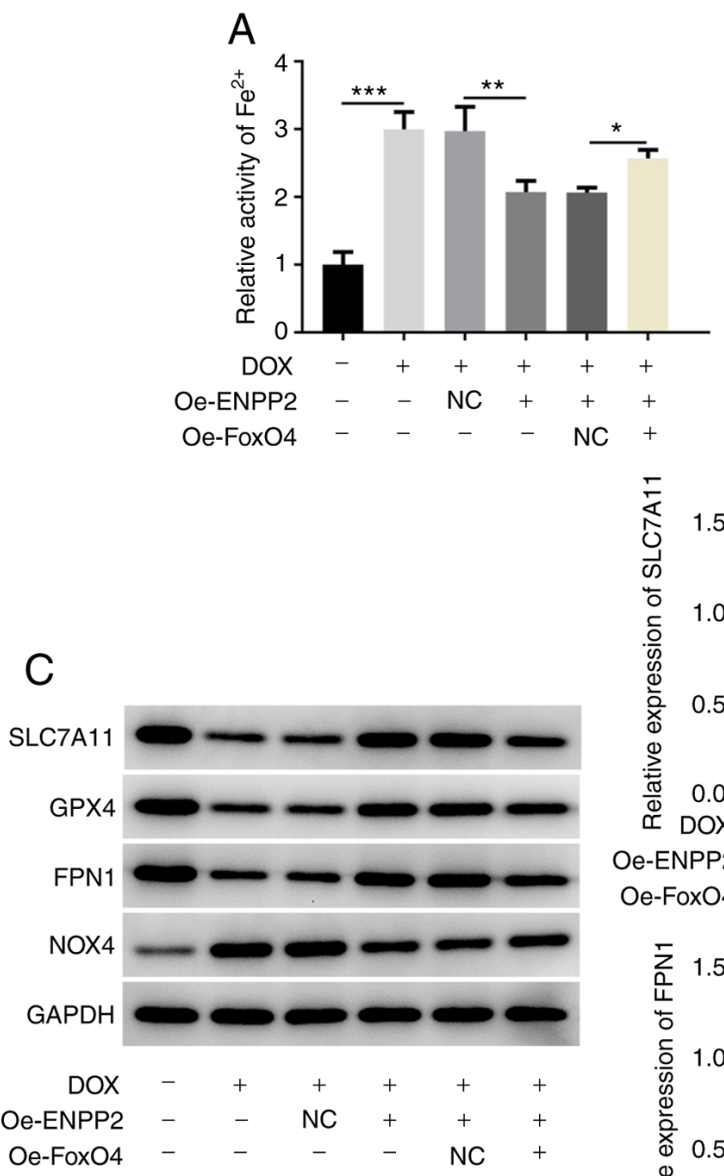
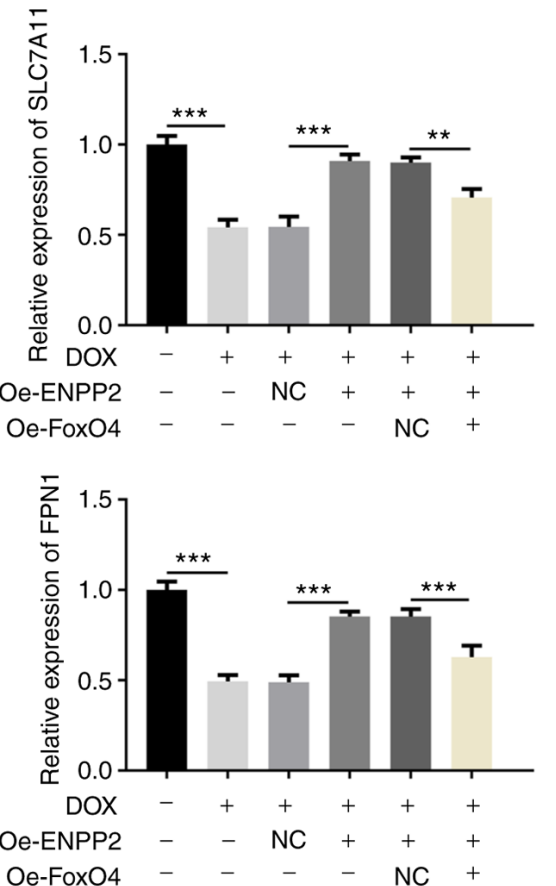
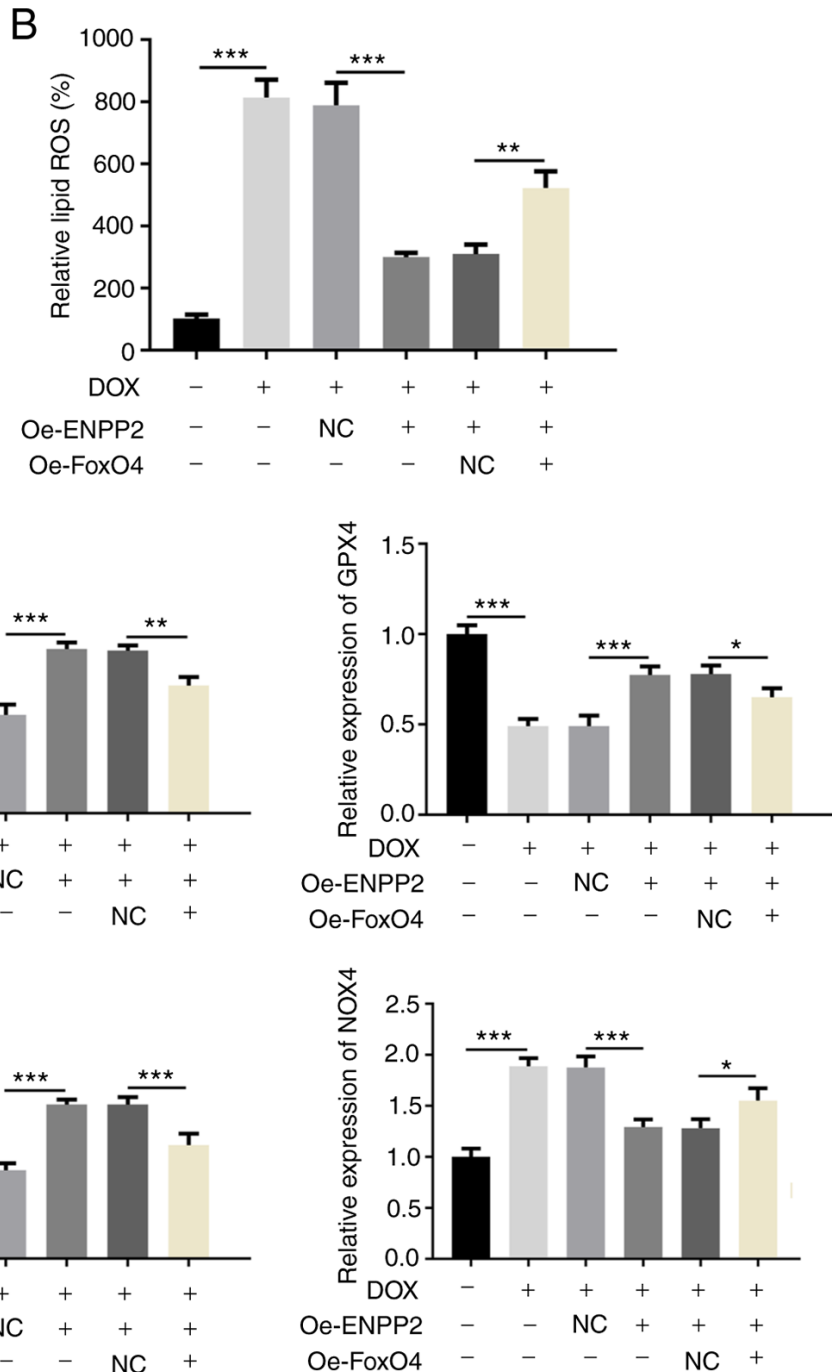

Figure 6. FoxO4 overexpression inhibits the effects of ENPP2 overexpression on DOX-induced ferroptosis in H9c2 cardiomyocytes. (A) H9c2 cells co-Oe FoxO4 and ENPP2 were exposed to DOX $(5 \mu \mathrm{M})$ for $24 \mathrm{~h}$ and cellular $\mathrm{Fe}^{2+}$ activity was measured using the Iron Assay kit. (B) The lipid ROS level was assessed. (C) The expression levels of the proteins involved in ferroptosis were measured using western blot analysis. ${ }^{*} \mathrm{P}<0.05,{ }^{* * *} \mathrm{P}<0.01$ and ${ }^{* * *} \mathrm{P}<0.001$. DOX, doxorubicin; ROS, reactive oxygen species; ENPP2, ectonucleotide pyrophosphatase/phosphodiesterase 2; Oe, overexpressing; NC, negative control; SLC7A11, solute carrier family 7 member 11; GPX4, GSH peroxidase 4; FPN1, ferroportin 1; NOX4, NADPH oxidase 4.
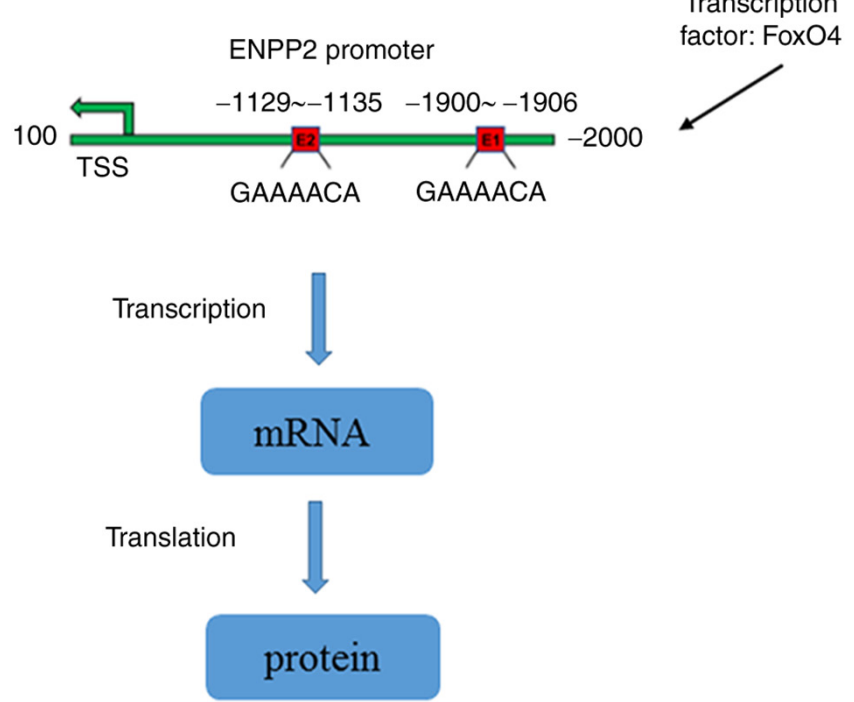

Figure 7. Transcription factor FoxO4 suppresses ENNP2 expression by binding to its promoter. ENPP2, ectonucleotide pyrophosphatase/phosphodiesterase 2.
However, there are some limitations to the present study that should be addressed, Firstly, in vivo experiments were not performed and the effects of FoxO4 overexpression on $\mathrm{H} 9 \mathrm{c} 2$ cells in the presence of DOX treatment were not investigated in the present study. Additionally, the absence of clinical data is another limitation of this study. Therefore, further experiments should be performed in future studies to support the present conclusions.

In conclusion, to the best of our knowledge, the current study was the first to investigate the role of ENNP2 in DOX-induced cardiomyocyte injury and its potential regulatory mechanisms. The results of this study demonstrated that FoxO4 could bind to the ENNP2 promoter to affect the post-transcriptional suppression of encoding mRNA and therefore negatively control the transcription of ENNP2 (Fig. 7), thereby regulating DOX-induced cardiotoxicity via the induction of ferroptosis. Specific therapies that target FoxO4/ENPP2 to reduce and increase FoxO4 and ENPP2 expressions, respectively, may provide potential approaches for ameliorating DOX-induced cardiotoxicity. 


\section{Acknowledgements}

Not applicable.

\section{Funding}

The present study was funded by the National Natural Science Foundation of China (grant no. 82060070) and the Science and Technology Research Project of Jiangxi Provincial Department of Education (grant no. 161453).

\section{Availability of data and materials}

The datasets used and/or analyzed during the current study are available from the corresponding author on reasonable request.

\section{Authors' contributions}

LH, YY and JL contributed to study conception or design. LH and YY performed the experiments and contributed to the acquisition of data. JC and PZ contributed to data analysis or interpretation. JL, LH and YY drafted the work and revised it critically for important intellectual content. All authors read and approved the final manuscript. LH and JL confirm the authenticity of all the raw data.

\section{Ethics approval and consent to participate}

Not applicable.

\section{Patient consent for publication}

Not applicable.

\section{Competing interests}

The authors declare that they have no competing interests.

\section{References}

1. Babiker HM, McBride A, Newton M, Boehmer LM Drucker AG, Gowan M, Cassagnol M, Camenisch TD, Anwer F and Hollands JM: Cardiotoxic effects of chemotherapy: A review of both cytotoxic and molecular targeted oncology therapies and their effect on the cardiovascular system. Crit Rev Oncol Hematol 126: 186-200, 2018.

2. Gupta SK, Garg A, Bär C, Chatterjee S, Foinquinos A, Milting H, Streckfuß-Bömeke K, Fiedler J and Thum T: Quaking inhibits doxorubicin-mediated cardiotoxicity through regulation of cardiac circular RNA expression. Circ Res 122: 246-254, 2018.

3. Tadokoro T, Ikeda M, Ide T, Deguchi H, Ikeda S, Okabe K, Ishikita A, Matsushima S, Koumura T, Yamada KI, et al: Mitochondria-dependent ferroptosis plays a pivotal role in doxorubicin cardiotoxicity. JCI Insight 5: e132747, 2020.

4. Fang X, Wang H, Han D, Xie E, Yang X, Wei J, Gu S, Gao F, Zhu N, Yin X, et al: Ferroptosis as a target for protection against cardiomyopathy. Proc Natl Acad Sci USA 116: 2672-2680, 2019.

5. Liu Y, Zeng L, Yang Y, Chen C, Wang D and Wang $\mathrm{H}$ Acyl-CoA thioesterase 1 prevents cardiomyocytes from Doxorubicin-induced ferroptosis via shaping the lipid composition. Cell Death Dis 11: 756, 2020.

6. Mou Y, Wang J, Wu J, He D, Zhang C, Duan C and Li B: Ferroptosis, a new form of cell death: Opportunities and challenges in cancer. J Hematol Oncol 12: 34, 2019.

7. Hirschhorn T and Stockwell BR: The development of the concept of ferroptosis. Free Radic Biol Med 133: 130-143, 2019.
8. Magkrioti C, Galaris A, Kanellopoulou P, Stylianaki EA, Kaffe E and Aidinis V: Autotaxin and chronic inflammatory diseases. J Autoimmun 104: 102327, 2019.

9. Zhao Y, Hasse S, Zhao C and Bourgoin SG: Targeting the autotaxin-Lysophosphatidic acid receptor axis in cardiovascular diseases. Biochem Pharmacol 164: 74-81, 2019.

10. Bai YT, Chang R, Wang H, Xiao FJ, Ge RL and Wang LS: ENPP2 protects cardiomyocytes from erastin-induced ferroptosis. Biochem Biophys Res Commun 499: 44-51, 2018.

11. Maiese K, Hou J, Chong ZZ and Shang YC: A fork in the path: Developing therapeutic inroads with FoxO proteins. Oxid Med Cell Longev 2: 119-129, 2009.

12. Zhu M, Goetsch SC, Wang Z, Luo R, Hill JA, Schneider J, Morris SM Jr and Liu ZP: FoxO4 promotes early inflammatory response upon myocardial infarction via endothelial Arg1. Circ Res 117: 967-977, 2015.

13. You J, Gao F, Tang H, Peng F, Jia L, Huang K, Chow K, Zhao J, Liu H, Lin Y and Chen J: A medicinal and edible formula YH0618 ameliorates the toxicity induced by Doxorubicin via regulating the expression of Bax/Bcl-2 and FOXO4. J Cancer 10: 3665-3677, 2019.

14. Wang Z, Zhang X, Tian X, Yang Y, Ma L, Wang J and Yu Y: CREB stimulates GPX4 transcription to inhibit ferroptosis in lung adenocarcinoma. Oncol Rep 45: 88, 2021.

15. Livak KJ and Schmittgen TD: Analysis of relative gene expression data using real-time quantitative PCR and the 2(-Delta Delta C(T)) method. Methods 25: 402-408, 2001

16. Jiang $Y$ and Zhang Q: Catalpol ameliorates doxorubicin-induced inflammation and oxidative stress in $\mathrm{H} 9 \mathrm{C} 2$ cells through PPAR- $\gamma$ activation. Exp Ther Med 20: 1003-1011, 2020.

17. Ye M, Zhang L, Yan Y and Lin $\mathrm{H}$ : Punicalagin protects $\mathrm{H} 9 \mathrm{c} 2$ cardiomyocytes from doxorubicin-induced toxicity through activation of Nrf2/HO-1 signaling. Biosci Rep 39: BSR20190229, 2019.

18. Hu C, Zhang X, Wei W, Zhang N, Wu H, Ma Z, Li L, Deng W and Tang Q: Matrine attenuates oxidative stress and cardiomyocyte apoptosis in doxorubicin-induced cardiotoxicity via maintaining AMPK $\alpha / U C P 2$ pathway. Acta Pharm Sin B 9: 690-701, 2019.

19. Shafei A, El-Bakly W, Sobhy A, Wagdy O, Reda A, Aboelenin O, Marzouk A, El Habak K, Mostafa R, Ali MA and Ellithy M: A review on the efficacy and toxicity of different doxorubicin nanoparticles for targeted therapy in metastatic breast cancer. Biomed Pharmacother 95: 1209-1218, 2017.

20. Abd-Rabou AA, Ahmed HH and Shalby AB: Selenium overcomes doxorubicin resistance in their nano-platforms against breast and colon cancers. Biol Trace Elem Res 193: 377-389, 2020.

21. Mikhail AS, Negussie AH, Pritchard WF, Haemmerich D, Woods D, Bakhutashvili I, Esparza-Trujillo J, Brancato SJ, Karanian J, Agarwal PK and Wood BJ: Lyso-thermosensitive liposomal doxorubicin for treatment of bladder cancer. Int J Hyperthermia 33: 733-740, 2017.

22. de Klerk E and 't Hoen PA: Alternative mRNA transcription, processing, and translation: Insights from RNA sequencing. Trends Genet 31: 128-139, 2015.

23. Shabalala S, Muller CJF, Louw J and Johnson R: Polyphenols, autophagy and doxorubicin-induced cardiotoxicity. Life Sci 180: 160-170, 2017.

24. Xiao B, Hong L, Cai X, Mei S, Zhang P and Shao L: The true colors of autophagy in doxorubicin-induced cardiotoxicity. Oncol Lett 18: 2165-2172, 2019.

25. Cappetta D, De Angelis A, Sapio L, Prezioso L, Illiano M, Quaini F, Rossi F, Berrino L, Naviglio S and Urbanek K: Oxidative stress and cellular response to doxorubicin: A common factor in the complex milieu of anthracycline cardiotoxicity. Oxid Med Cell Longev 2017: 1521020, 2017.

26. Wu C, Zhao W, Yu J, Li S, Lin L and Chen X: Induction of ferroptosis and mitochondrial dysfunction by oxidative stress in PC12 cells. Sci Rep 8: 574, 2018.

27. Latunde-Dada GO: Ferroptosis: Role of lipid peroxidation, iron and ferritinophagy. Biochim Biophys Acta Gen Subj 1861: 1893-1900, 2017.

28. Seibt TM, Proneth B and Conrad M: Role of GPX4 in ferroptosis and its pharmacological implication. Free Radic Biol Med 133: 144-152, 2019.

29. Luo LF, Guan P, Qin LY, Wang JX, Wang N and Ji ES: Astragaloside IV inhibits adriamycin-induced cardiac ferroptosis by enhancing Nrf2 signaling. Mol Cell Biochem 476: 2603-2611, 2021.

30. Liu T, Jiang L, Tavana O and Gu W: The deubiquitylase OTUB1 mediates ferroptosis via stabilization of SLC7A11. Cancer Res 79: 1913-1924, 2019. 
31. Wang Z, Ding Y, Wang X, Lu S, Wang C, He C, Wang L, Piao M, Chi G, Luo Y and Ge P: Pseudolaric acid B triggers ferroptosis in glioma cells via activation of Nox4 and inhibition of xCT. Cancer Lett 428: 21-33, 2018.

32. Xu H, Yu W, Sun S, Li C, Zhang Y and Ren J: Luteolin attenuates doxorubicin-induced cardiotoxicity through promoting mitochondrial autophagy. Front Physiol 11: 113, 2020.

33. Zhou B, Liu J, Kang R, Klionsky DJ, Kroemer G and Tang D: Ferroptosis is a type of autophagy-dependent cell death. Semin Cancer Biol 66: 89-100, 2020.

34. Liu J, Kuang F, Kroemer G, Klionsky DJ, Kang R and Tang D Autophagy-dependent ferroptosis: Machinery and regulation. Cell Chem Biol 27: 420-435, 2020.
35. Oteiza A and Mechti N: FoxO4 negatively controls Tat-mediated HIV-1 transcription through the post-transcriptional suppression of Tat encoding mRNA. J Gen Virol 98: 1864-1878, 2017.

36. Yu L, Zhang W, Huang C, Liang Q, Bao H, Gong Z, Xu M, Wang $\mathrm{Z}$, Wen $\mathrm{M}$ and Cheng X: FoxO4 promotes myocardial ischemia-reperfusion injury: The role of oxidative stress-induced apoptosis. Am J Transl Res 10: 2890-2900, 2018.

This work is licensed under a Creative Commons Attribution-NonCommercial-NoDerivatives 4.0 International (CC BY-NC-ND 4.0) License. 